\title{
Optical cellulose fiber made from regenerated cellulose and cellulose acetate for water sensor applications
}

\author{
H. Orelma • A. Hokkanen • I. Leppänen · K. Kammiovirta • M. Kapulainen • \\ A. Harlin
}

Received: 11 April 2019/Accepted: 20 November 2019/Published online: 25 November 2019

(C) The Author(s) 2019

\begin{abstract}
In this study an optical cellulose fiber for water sensoring was prepared by using a sequential preparation strategy. The core of the fiber was prepared from dissolved cellulose, in [EMIM]OAc, which was dry-wet spun into water. The cladding layer on the cellulose core was produced by coating a layer of cellulose acetate, dissolved in acetone, using a filament coater. The chemical and optical properties of both regenerated cellulose and cellulose acetate were studied from cast films using ultraviolet-visible and Fourier-transform infrared spectroscopy measurements. Regenerated cellulose film was observed to absorb UV light, passing the visible light wavelengths. Cellulose acetate film was observed to pass the whole light wavelength range. The mechanical strength and topography of the prepared optical cellulose fiber were
\end{abstract}

investigated through tensile testing and SEM imaging. The mechanical performance of the fiber was similar to previously reported values in the literature (tensile strength of $120 \mathrm{MPa}$ ). The prepared optical fiber guided light in the range of 500-1400 nm. The attenuation constant of the cellulose fiber was observed to be $6.3 \mathrm{~dB} / \mathrm{cm}$ at $1300 \mathrm{~nm}$. The use of prepared optical cellulose fiber in a water sensor application was demonstrated. When the fiber was placed in water, a clear attenuation in the light intensity was observed. The studied optical fiber could be used in sensor applications, in which easy modifiability and high thermal resistance are beneficial characteristics.

Electronic supplementary material The online version of this article (https://doi.org/10.1007/s10570-019-02882-3) contains supplementary material, which is available to authorized users.

H. Orelma $(\bowtie) \cdot$ I. Leppänen · K. Kammiovirta .

A. Harlin

High Performance Fiber Products, VTT Technical

Research Centre of Finland Ltd, Biologinkuja 7,

02044 Espoo, Finland

e-mail: hannes.orelma@vtt.fi

\section{A. Hokkanen · M. Kapulainen}

Photonics Integration, VTT Technical Research Centre of

Finland Ltd, Tietotie 3, 02044 Espoo, Finland 
Graphic abstract Coaxial cellulose acetate-regenerated cellulose fiber for transporting light in sensor optical fiber sensor applications.
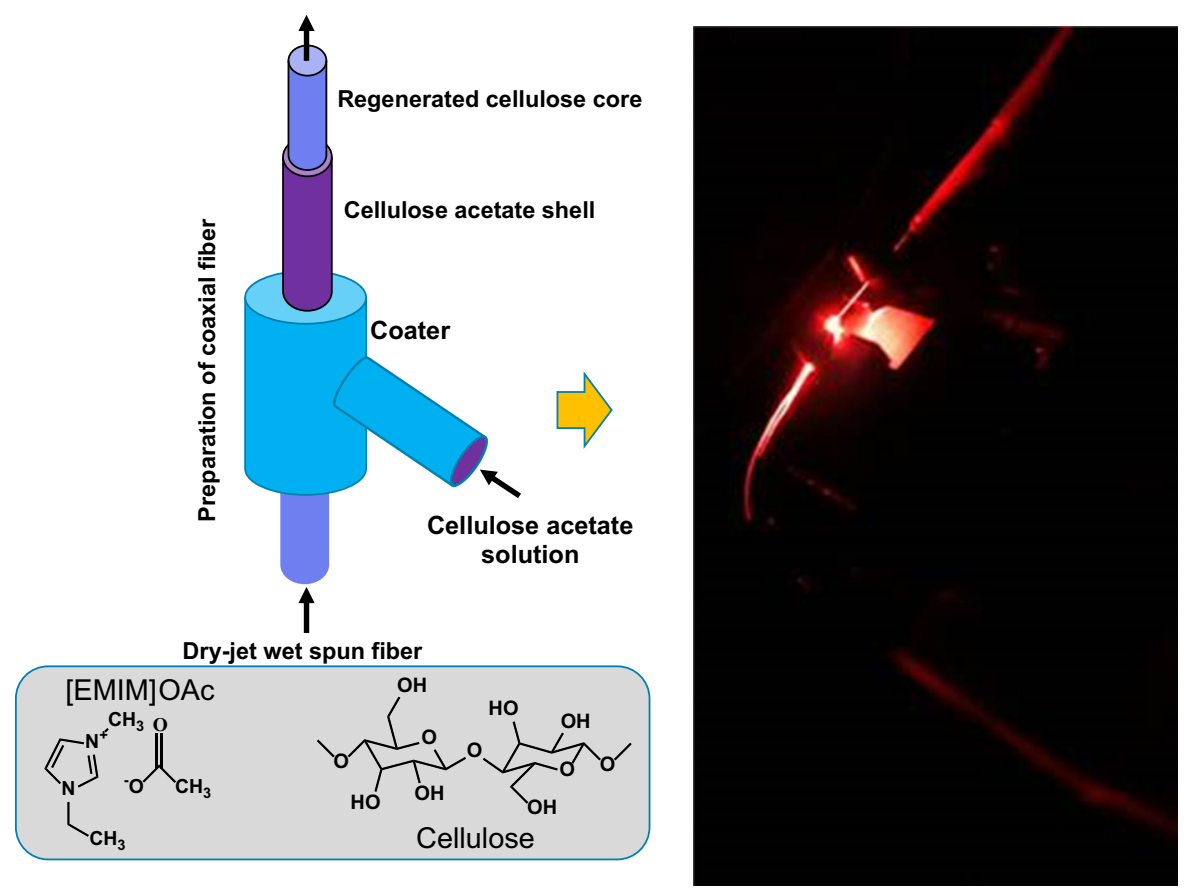

Keywords Cellulose $\cdot$ Cellulose acetate $\cdot$ Optical fiber - Coaxial fiber - Water sensor

\section{Introduction}

Optical fibers are present in our daily life in multiple applications from data communication to sensors. Typically, optical fibers are made from glass and plastic (Senior and Jamro 2009). From these, glassbased fibers have excellent data transportation properties over long distances (Sanghera et al. 2002), whereas plastic-based fibers have been widely used in optical sensors and lighting applications (Zubia and Arrue 2001). Optical fibers are coaxial structures that transport light by utilizing the dielectric waveguide behavior. This idea was first theorized by Hondros and Debye (1910) and experimentally verified by Schiever (1920). In an ideal optically transparent material, higher refractive index material is covered with a lower refractive index material. Then the core guides the light through without scattering and the total internal reflection in the interface between core and cladding keeps the light inside the core. As early as 1965, Kao and Hockham demonstrated that it was possible to have less than $20 \mathrm{~dB} / \mathrm{km}$ attenuation with glass silica fibers and that such fibers were suitable for communication transmission (Sanghera et al. 2002). Moreover, the best optical fibers today have less than $0.1 \mathrm{~dB} / \mathrm{km}$ attenuation. In real life, all materials attenuate light due to the presence diffraction from interfaces, chromophoric substances or uneven geometries. The dimensions and refractive index difference influence the electric field modes of the optical fiber (Born and Wolf 2013). Single-mode operation can be achieved typically below $10 \mu \mathrm{m}$ core size. Multi-mode operation takes place with the diameter of the core above approximately $10 \mu \mathrm{m}$, though both refractive index and wavelength also influence the diameter limit. In general, the used materials, dimensions and optical connections influence light transmission in optical fibers.

The global consumption of fossil-based materials has created an increasing need to develop more sustainable materials to prevent carbon dioxide 
emissions to the atmosphere and plastic pollution on land and at sea (Schmalensee et al. 1998; Browne et al. 2011). In this context, plant-based cellulose will play a significant role because of its high modification potential, renewability and biodegradability (Schlamadinger and Marland 1996). Regenerated cellulose materials are produced by first dissolving purified cellulose fibers in a suitable solvent and then regenerating the dissolved material back into solid cellulose in a suitable antisolvent (Liebert 2010). Cellulose solvents can be classified as non-derivatizing and derivatizing cellulose solvents (Heinze and Koschella 2005; Lindman et al. 2010). Non-derivatizing cellulose solvents are direct cellulose solvents (for example, NMMO, DMAc/LiCl, ionic liquids) that do not change in the chemistry of cellulose, whereas derivatizing solvents (for example, $\mathrm{NaOH} / \mathrm{urea}$ and viscose) graft cellulose in order to make them soluble in liquid media followed by solidification in a suitable antisolvent or evaporation of solvent medium. The dissolved cellulose can be chemically modified in a wide range of substitutes in cellulose solvent-producing cellulose derivative materials (examples of these include cellulose acetate, carboxymethyl cellulose, methyl cellulose) (Klemm et al. 1998; Liebert and Heinze 2005). Moreover, selection of the substituent influences the refractive index of the cellulose material making it possible to form interface pairs from cellulose materials (Mark 2007). Polymeric cellulose materials are typically transparent due to the lack of reflective interfaces and the low chemical absorbance of light in the range of visible light.

Glass- or plastic-based optical fibers are excellent in communication applications although it is difficult to modify the material itself for sensor applications. Water and gas do not penetrate inside glass or polymer material and react with the material itself, but porous cellulose material takes water and gas inside and has the ability to react with them. For that reason glass and polymer fiber sensors have typically an active coating on their surface. Cellulose fiber is accordingly active material itself and it could be even biodegradable. Andrade et al. measured biomolecules on silica optical fiber with fluorescence labeled immunoassay (Andrade et al. 1985). Evanescent wave had around a $200 \mathrm{~nm}$ penetration depth into the liquid sample. Sekimoto et al. coated silica fiber with platinum- or palladium-supported tungsten oxide and demonstrated a hydrogen gas sensor (Sekimoto et al. 2000). They showed evanescent wave absorption in tungsten oxide between a $400-800 \mathrm{~nm}$ wavelength range. It is also possible to make holes inside optical fibers, so-called capillary fibers or photonic crystal fibers. Dhadwal et al. (2004) made fluorescence based nucleic acid biosensor inside glass capillary fiber. They measured $30 \mathrm{pg} / \mathrm{ml}$ DNA molecule concentration. Wu et al. developed a photonic crystal fiber-based refractive index sensor (Wu et al. 2009). They filled one $3.6 \mu \mathrm{m}$ hole with a liquid sample and achieved a $4.6 \times 10^{-7}$ detection limit for refractive index. Gas sensing using photonic crystal fibers was presented by Ritari et al. (2004). They measured the absorption lines of acetylene, hydrogen cyanide, methane and ammonia in a $10-11.6 \mu \mathrm{m}$ hole. Also, plastic optical fibers have been previously used in many sensor applications including water sensoring (Peters 2011). A sheet of cellulose acetate was connected to the end of a plastic optical sensor and the swelling of the sheet in humid air was monitored (Xu et al. 2013). A citrate-based flexible optical sensor has been developed for medical applications for delivering light into the body (Shan et al. 2017). Cellulose materials have similar properties to the given materials since they are also body compatible already used in medical applications (Sindhu et al. 2014). The use of optical fibers in medical applications has been previously reviewed (Nazempour et al. 2018). Biodegradable microstructured optical fiber has previously been prepared from cellulose acetate butyrate (Dupuis et al. 2007). However, this is not a typical optical fiber and it contained a three layer coaxial structure made from two on the other placed tubes with an air core. In general, cellulose has not been studied as optical fiber due assumption of cellulose as improper optical material, while it is inherently transparent polymer having wide variety of derivate with different refractive indexes.

In this study, we investigated the preparation of an optical cellulose fiber from regenerated cellulose (higher refractive index) and cellulose acetate (lower refractive index). The optical cellulose fiber was prepared using a two-phase process in which the regenerated cellulose core was produced from [EMI$\mathrm{M}] \mathrm{OAc}$ by using dry-jet wet spinning and water as a coagulant (Scheme 1). The shell was produced by coating the cellulose core with cellulose acetate dissolved in acetone. The developed cellulose-based optical fiber could be used in sensoring and medical applications, in which the easy tenability, high 


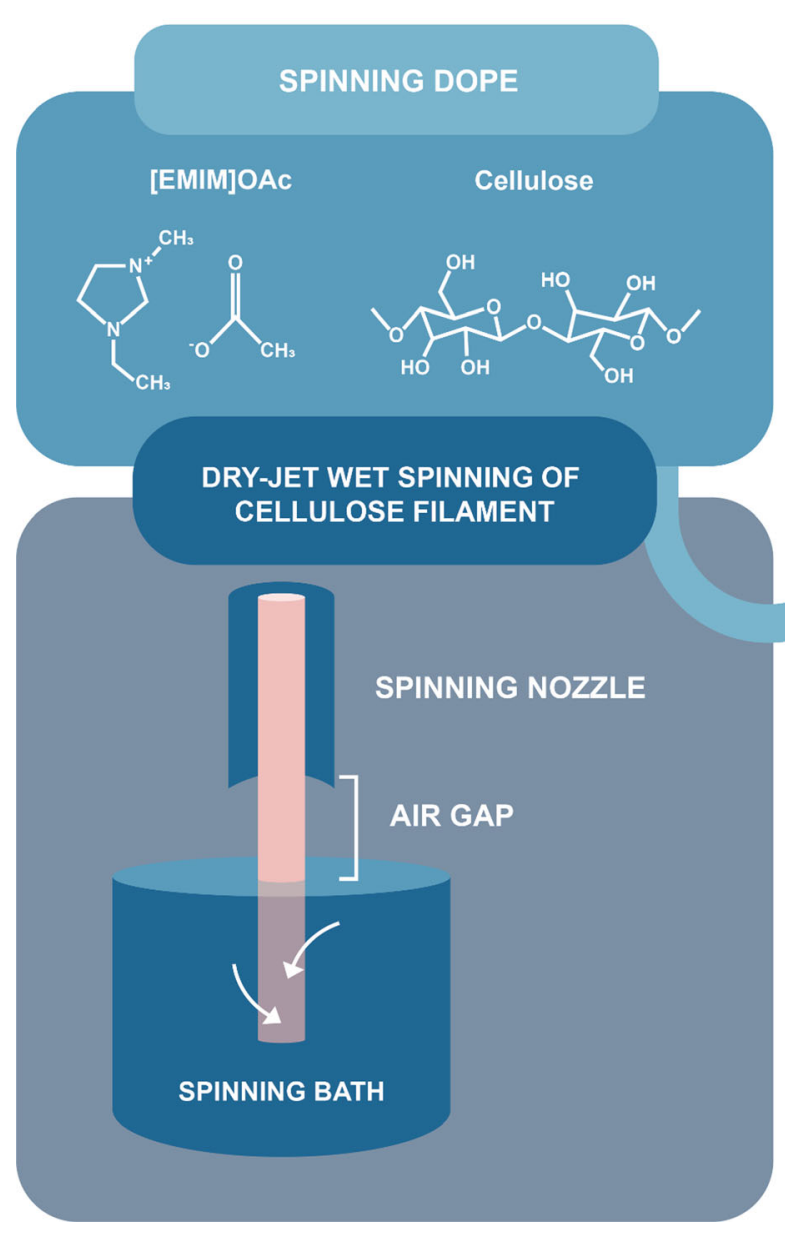

Scheme 1 The manufacturing process of optical cellulose fiber. Cellulose was first dissolved in [EMIM]OAc and dry-jet wet spun into water. The water was then evaporated from the filament at room atmosphere. The optical cladding was

temperature resistance and biocompatibility could be beneficial properties.

\section{Experiment section}

Materials

The cellulose source was bleached softwood kraft pulp (Metsä Fiber Äänekoski pulp mill, Finland). The pulp was air-dried and then dry crushed in a Waring blender to rupture fiber flocs and separate single fibers. 1-Ethyl-3-methylimidazolium acetate [EMIM]OAc was purchased from Ionic Liquids Technologies GmbH Heilbronn, Germany, and was used as

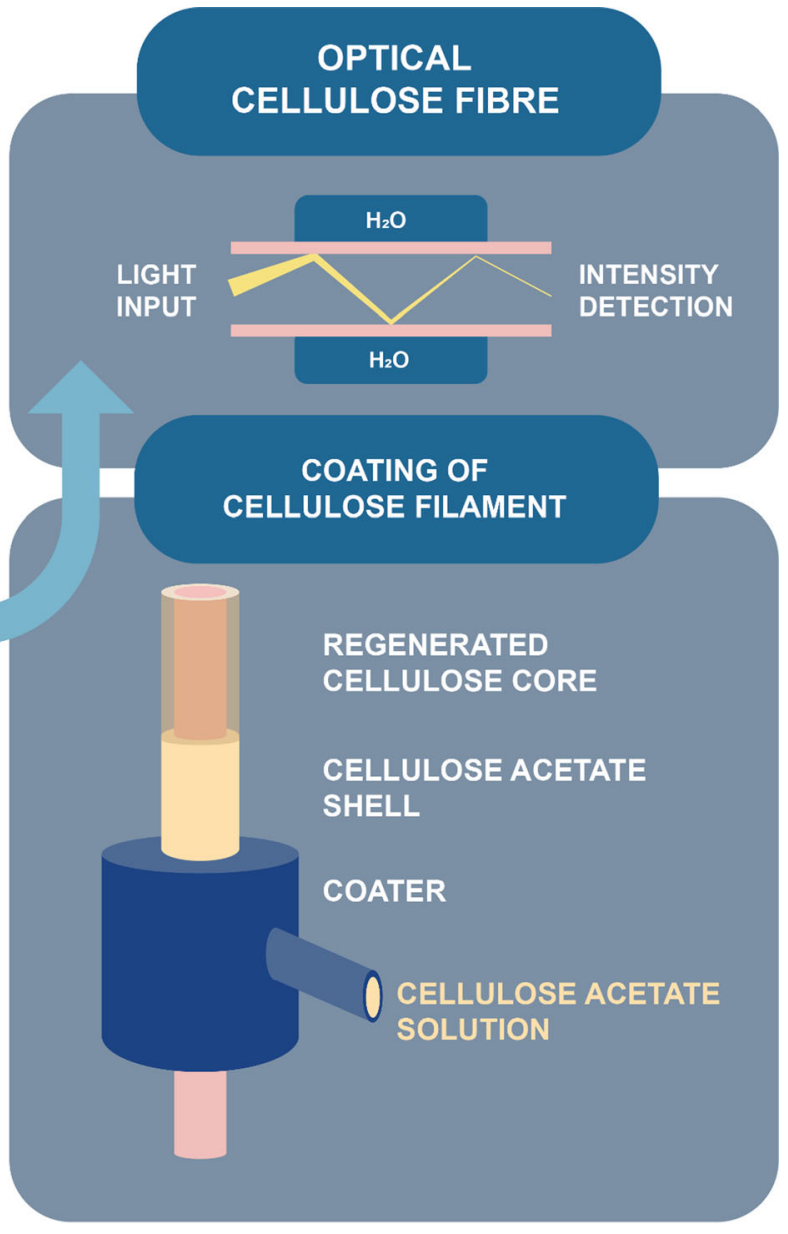

produced by coating the cellulose core with cellulose acetate dissolved in acetone. The prepared optical cellulose fiber was connected to a light source and its optical properties in air and water were then studied

received. Cellulose acetate (DS 3, Mw 30 $000 \mathrm{~g} / \mathrm{mol}$ ) was obtained from Sigma Aldrich (Finland). All other chemicals were laboratory grade. All water used in the study was purified using a Milli-Q device.

Methods

\section{Dissolution of cellulose}

$2.5 \mathrm{~g}$ of cellulose was dissolved in $47.5 \mathrm{~g}$ of [EMIM]AcO at $130{ }^{\circ} \mathrm{C}$ until complete dissolution occurred (approximately $150 \mathrm{~min}$.). The dry consistency of the final solution was $5 \mathrm{wt} \%$ and dissolution was carried out using a System Reaction Carousel 
Station (Radley, Saffron Walden, United Kingdom) with $100 \mathrm{ml}$ reaction flasks. Within the dissolution medium, the solution was continuously mixed with a slowly rotating blade. The dissolved solution was stored at room conditions prior to spinning into a filament.

\section{Preparation of optical fiber from dissolved cellulose}

The core filament was prepared from the cellulose dope using a laboratory-scale wet-jet spinning device equipped with a spinning nozzle. A $10 \mathrm{ml}$ plastic syringe was filled by the dissolved cellulose and then passed through a nozzle (diameter and length $0.41 \mu \mathrm{m}$ and $31.75 \mathrm{~mm}$, respectively) into a water coagulation bath. A constant spinning rate of $0.5 \mathrm{ml} / \mathrm{min}$ was used. After spinning, the filaments were kept in water for at least two hours and were then dried under tension at room temperature and ambient humidity.

The cladding layer was prepared on a core filament using a self-made coater. $25 \mathrm{~g}$ of cellulose acetate was dissolved in $25 \mathrm{~g}$ of acetone using a laboratory mixer. The dissolved CA was placed into the coater and the premade filament was drawn through the self-made filament coater. The acetone was evaporated from the coated filament at room atmosphere. The thickness of the cellulose acetate layer was governed by the diameter of the output hole.

\section{Preparation of films from regenerated cellulose and cellulose acetate}

The regenerated cellulose film was produced from cellulose dissolved in [EMIM]OAc that was coated on a glass surface using an Erichsen Coatmaster 510 film applicator with a $500 \mu \mathrm{m}$ rod. The dissolved cellulose layer on the glass plate was regenerated in water for $1.5 \mathrm{~h}$. The regenerated cellulose film was dried between absorbent papers at RT for 3 days. Average film thickness was $74 \mu \mathrm{m}$.

Cellulose acetate film was produced from cellulose acetate (CA) dissolved in acetone (consistency $20 \mathrm{wt} \%)$. The dissolved cellulose acetate was coated on a transparent plastic film using the Erichsen Coatmaster 510 film applicator with a $500 \mu \mathrm{m}$ rod. The acetone was evaporated at RT overnight. Average film thickness was $65 \mu \mathrm{m}$.

\section{UV-VIS measurements}

The optical properties of the regenerated cellulose and cellulose acetate films were investigated using a Lambda 900 UV/VIS/NIR spectrometer (PerkinElmer, USA) with a film holder. The samples were cut into pieces of approximately $1 \times 4 \mathrm{~cm}^{2}$. UV spectra were measured using a light wavelength range of $250-800 \mathrm{~nm}$ with a $1 \mathrm{~nm}$ measurement resolution. The intensity curves were normalized by the film thicknesses in order to remove the thickness effect from the graphs. At least five repetitions per sample were conducted.

\section{Fourier transform infrared (FTIR) microscopy analyses}

FTIR spectroscopy was used to study the regenerated cellulose and cellulose acetate materials from films. The characterization was performed using a Thermo Scientific Nicolet iS50 FT-IR spectrometer with an ATR diamond (Thermo Scientific, USA). All spectra were obtained from 32 scans with a resolution of $4 \mathrm{~cm}^{-1}$ and absorption mode by using a wavelength range from 400 to $4000 \mathrm{~cm}^{-1}$. At least three repetitions per sample were conducted. The FTIR spectra of the used materials are shown in the Figure S1 supporting information.

\section{SEM imaging of optical cellulose fibers}

SEM imaging was performed using a Merlin FE-SEM (Carl Zeiss NTS GmbH, Germany) with a gold sputter coating. First, all samples were mounted onto aluminum specimen stubs with double-sided carbon adhesive discs. The samples were then coated with a thin layer of gold to prevent charging of sample surfaces under an electron beam. Imaging was performed using $5 \mathrm{keV}$ electron energy with the secondary electron detector. The size of all SEM images was fixed at $2048 \times 1536$ pixels.

\section{Mechanical properties of optical cellulose fibers}

The mechanical properties of optical cellulose fibers were analyzed using a Lloyd LS5 materials testing machine (AMETEK measurement and calibration technologies, USA) at $23{ }^{\circ} \mathrm{C}$ and $50 \% \mathrm{RH}$ with a load cell of $100 \mathrm{~N}$. All samples were allowed to stabilize in 
standard conditions overnight as a minimum. The initial grip distance was kept at a constant $40 \mathrm{~mm}$ and the rate of the grip separation was a constant $10 \mathrm{~mm} /$ min. The thickness of each fiber sample was measured using a digital caliber from 5 different staples. The average thickness was used in the calculations. All samples were replicated at least seven times.

\section{Light transportation and attenuation constant of optical cellulose fibers}

The optical transparency of the produced optical cellulose fibers was measured using an Ocean Optics HK-2000-HP halogen lamp and an Ando AQ-6315A Optical Spectrum Analyzer (OSA) (Fig. 1a, b). The measured wavelength range was $350-1750 \mathrm{~nm}$ and the measurement resolution of OSA was $10 \mathrm{~nm}$. The attenuation spectrum and constant at $1300 \mathrm{~nm}$ were calculated from the OSA signal. Attenuation of the longest cellulose fiber sample and sensoring of water by the prepared optical cellulose fiber were studied using a Thorlabs' fiber-coupled superluminescent diode (SLD) light source. The center wavelength of this SLD light source is $1310 \mathrm{~nm}$ and bandwidth is $85 \mathrm{~nm}$.

Input and output fibers were glass silica fibers from Thorlabs. The fibers had $105 \mu \mathrm{m}$ core and $125 \mu \mathrm{m}$ cladding diameters. Input and output fibers were chosen that were smaller than cellulose fibers in order to avoid direct light coupling from the input to output fibers, because the cellulose fibers were just a few centimeters long. For the same reason, the longer cellulose fibers were also bent slightly during the measurements, to show that they can waveguide light.

\section{Results and discussion}

Optical properties of cellulose materials

The optical properties of regenerated cellulose and cellulose acetate were analyzed from cast films by the UV-VIS absorbance measurements. To the naked eye, both materials appear highly transparent. However, when the given materials were analyzed using UVVIS, a clear difference between the cellulose materials was observed (Fig. 2). In the UV range (250-400 nm), the regenerated cellulose absorbed significantly more light than the cellulose acetate. It has been reported that glucose exhibits a broad UV absorption band at $270 \mathrm{~nm}$ (Kaijanen et al. 2015). The absorption spectrum of regenerated cellulose decreased monotonically and reached the plateau at $600 \mathrm{~nm}$. Whereas, the spectrum of cellulose acetate was almost flat in the entire UV-visible wavelength range. The observed behavior is similar to what has previously been reported in the literature for pure and deacetylated cellulose acetate (Rowen et al. 1947). The observed data also correlates with a study in which the optical transparency of ZnS-sodium carboxymethyl cellulose nanocomposite film was examined using UV-Vis spectroscopy (Luna-Martínez et al. 2011).
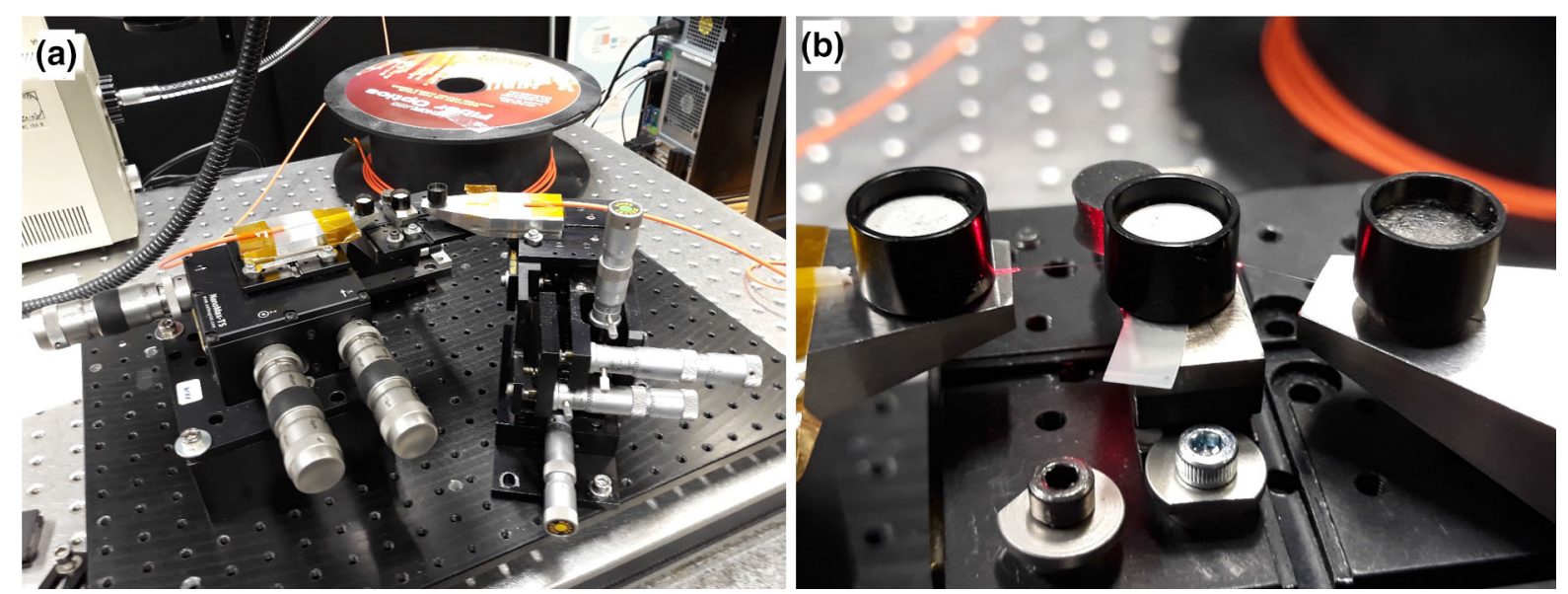

Fig. 1 Measurement setup for optical fibers. a Micromanipulators for measuring optical transparency and b light coupling for measuring the attenuation constant 


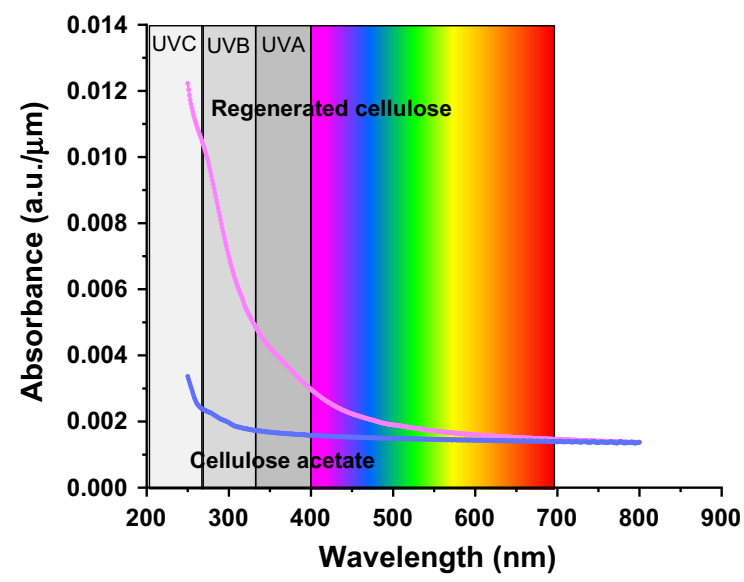

Fig. 2 UV-VIS spectra of cellulose-based films. The absorbance values are normalized by the film thickness in order to remove the effect of thickness variations between samples

Preparation of optical cellulose fibers

The optical cellulose fibers were produced using a sequential manufacturing method (Scheme 1). The core of the optical fiber was produced by dry-jet wet spinning cellulose dissolved in [EMIM]OAc through a nozzle into water. The production technology for manufacturing cellulose filaments through IL dissolution and dry-jet wet spinning has already been reported in the literature (Hauru et al. 2014). The spinning produced a uniform monofilament that was purified from IL through multiple water washes. The filament was evacuated from water and allowed to dry under tension. This also orientates the cellulose in the filament. The shape of the core was rather circular, but we did not measured the changes in the shape factor along the fiber, which may influence to the light wave guiding properties of the optical fiber. The cellulose acetate shell (cladding) was produced by drawing the premade cellulose filament through a cellulose acetate dissolved in acetone. The film thickness was fixed using a lab-made filament coater. An SEM image of the optical cellulose fiber is shown in Fig. 3a. From the SEM image it can be seen that the produced fiber contained a uniform cellulose acetate layer on the cellulose core. It can be assumed that the orientation of the cellulose acetate layer is low due to applied slow coating speed. The diameter of the filament was approximately $210 \mu \mathrm{m}$ and the cellulose acetate layer thickness was $3.40 \pm 0.20 \mu \mathrm{m}$. It appears that cellulose acetate adheres rather well to regenerated cellulose since no open cavity between the cladding and core was observed (Fig. 3b). However, the cut edge shows that the cellulose acetate layer was released from the surface when the SEM sample was prepared. We have previously shown that cellulose acetate adheres strongly to regenerated cellulose fabric (Tenhunen et al. 2018). However, with a dense filament it is possible that cellulose acetate is unable to deeply penetrate in the regenerated cellulose core. In addition, from the SEM images cannot be studied the nanoscale evenness of the cladding layer. The cut edge was not smooth, which means that the cutting of optical cellulose filaments requires more research as the uneven optical connections result in optical losses (Klein et al. 2010).

The mechanical properties of the produced optical fiber were measured using a universal tensile tester. The Young's modulus and tensile strength were $5.4 \pm 0.2 \mathrm{GPa}$ and $129 \pm 5 \mathrm{MPa}$, respectively. The values measured here were comparable to values reported previously (Hauru et al. 2014). The strain at break (conditioned) was $21 \pm 3 \%$. The reason for the large strain at break was that the fiber was not oriented with drawing during the fiber spinning. The properties of the cellulose core can be controlled by the spin dope and spinning conditions used (Hauru et al. 2014; Michud et al. 2016).

Light transporting properties of optical cellulose fiber

The prepared optical cellulose fiber was connected to a micromanipulator setup of optical fibers and the light transmission was measured as a function of light wavelength from four different length specimens (15, 24, 38 and $50 \mathrm{~mm}$ ) (Fig. 4a). Optical cellulose fiber mainly guides light in the wavelength range of 500-1400 $\mathrm{nm}$. As an example, the intensity difference of the $15 \mathrm{~mm}$-long sample at $1300 \mathrm{~nm}$ had a $25 \mathrm{~dB}$ lower signal than the input fiber to output fiber reference. The measurements show one peak maximum at the $1300 \mathrm{~nm}$ range, which is one of the telecommunication wavelength ranges. This makes it possible to use inexpensive telecommunication light sources and detectors for sensor applications. In the UV range $(\lambda<400 \mathrm{~nm})$, the light intensity was totally attenuated. The results correlated well with the UVVIS measurements (Fig. 2a), in which the UVscreening effect of pure cellulose was observed. In 

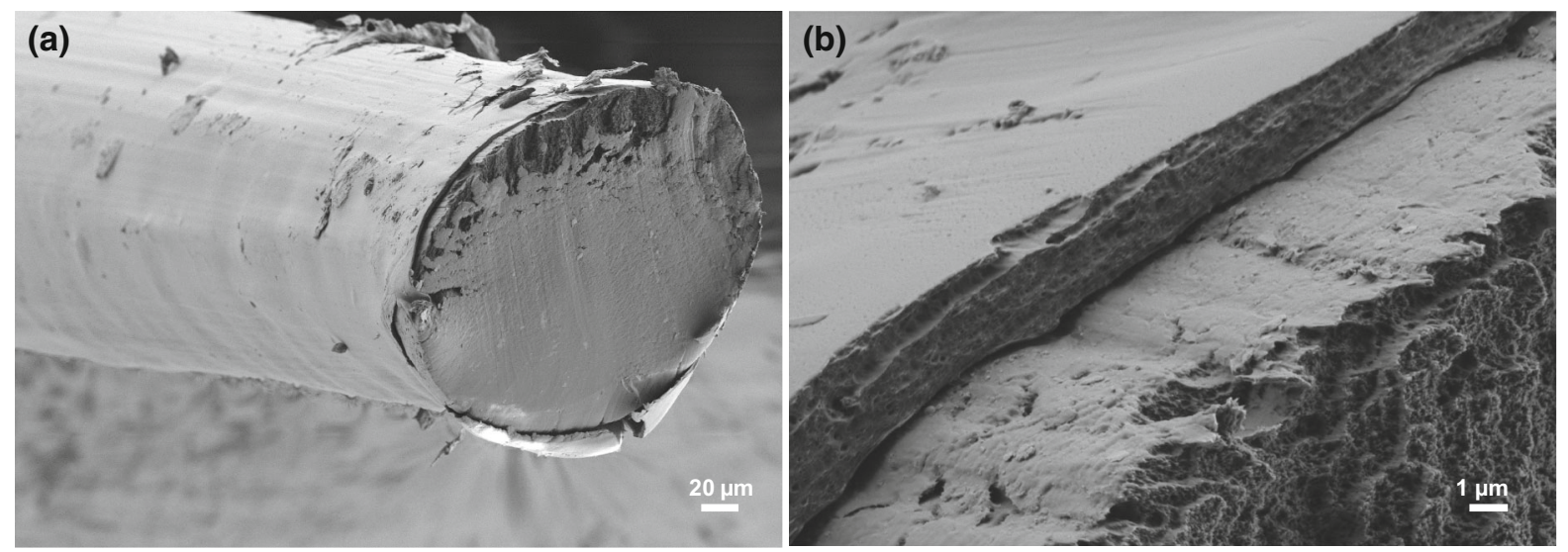

Fig. 3 SEM images of the cross-section of produced coaxial fiber. Image magnifications were $\mathbf{a} 269 \times$ and $\mathbf{b} 5890 \times$. The defects seen in the cut section were formed when cutting the fiber for SEM imaging
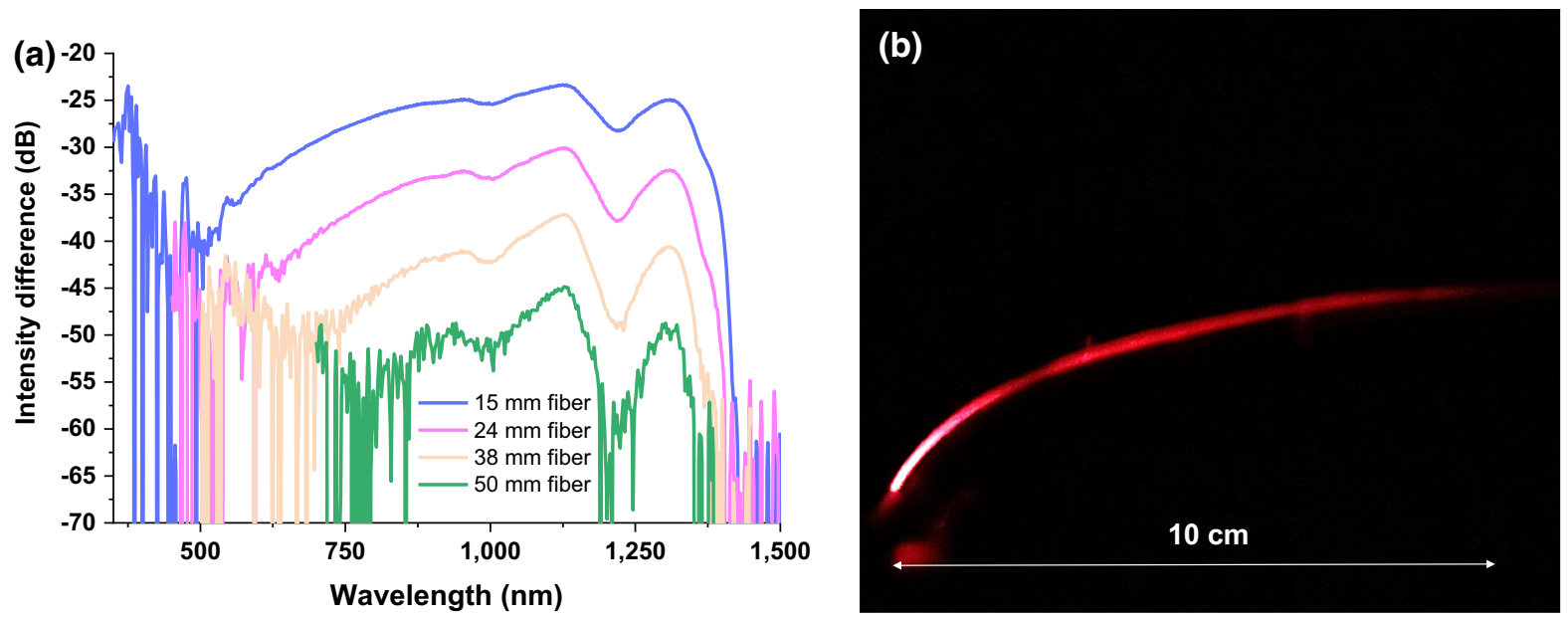

Fig. 4 Intensity difference of light as a function of light wavelength (a). The light source was a halogen lamp. Photo image of the investigated optical fiber, transmitting red light through a $10 \mathrm{~cm}$-long sample (b)

general, it can be concluded that the optical cellulose fiber is best suited to transmitting light in the infrared (IR) range $(800<\lambda<1300 \mathrm{~nm})$. The usability of optical cellulose fiber in lighting solutions was visually investigated by connecting a red laser to the end of the optical fiber (Fig. 4b). It can be seen that the light from red laser passed through the $10 \mathrm{~cm}$-long specimen but the red color leaking from the cellulose acetate cladding indicates that the cladding layer was not fully capable of keeping the scattered light in the core. Light is scattered from the cellulose fibers in the core and this can be improved with better cellulose fiber alignment in the core. Cladding modification with lower refractive index could only slightly improve this scattering loss.
The attenuation spectrum as a function of light wavelength was calculated from intensity difference values in 15, 24 and 38 mm-long specimens (Fig. 5a). The intensity difference value for each wavelength was linearly fitted to 15,24 and $38 \mathrm{~mm}$-long specimens. Attenuation was calculated from the slope of this linear fitting. The attenuation constant minimum of $5.9 \mathrm{~dB} / \mathrm{cm}$ was found at $1130 \mathrm{~nm}$. In general, the attenuation constant was under $10 \mathrm{~dB} / \mathrm{cm}$ in the 750-1350 $\mathrm{nm}$ range, in which the $\mathrm{R}^{2}$ squared value for linear fitting was over 0.96. The attenuation constant in the $1300 \mathrm{~nm}$ range as a function of the length of the optical fiber was also measured (Fig. 5b). The longest fiber, $76 \mathrm{~mm}$, was measured using an SLD light source to maximize the fiber length. It was 

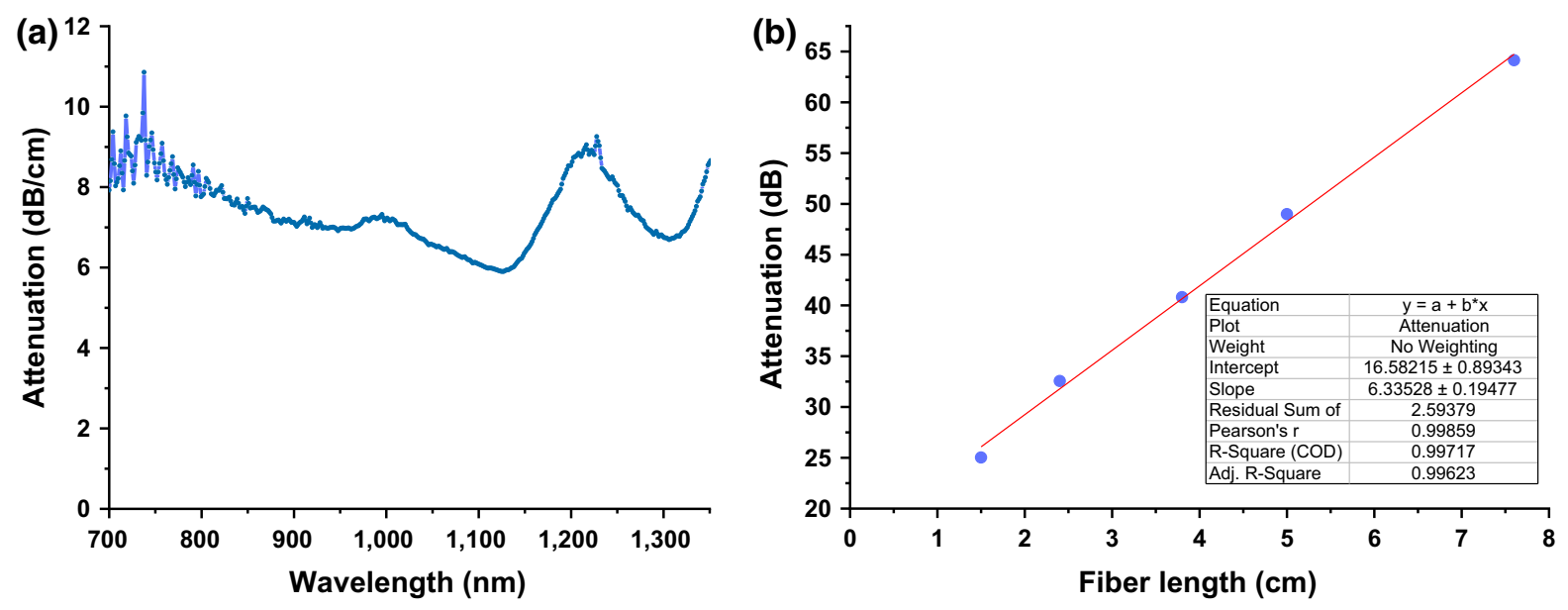

Fig. 5 Attenuation spectrum of the optical cellulose fiber as a function of light wavelength (a). Attenuation of the optical cellulose fiber at $1300 \mathrm{~nm}$ as a function of sample length (b). The fitting parameters are shown in the figure insert

observed that the attenuation increased linearly as a function of the length of the optical fiber. By fitting a linear line, an attenuation constant of $6.3 \mathrm{~dB} / \mathrm{cm}$ at $1300 \mathrm{~nm}$ was achieved. Moreover, the coupling attenuation was calculated to be $16.6 \mathrm{~dB}$, indicating a high coupling loss between the cellulose fiber and the measurement fiber. A part of this coupling attenuation stems from measurement fibers, because the core diameter of the cellulose fiber was $210 \mu \mathrm{m}$ that was $2 \times$ from the core diameter of the measurement fibers $(105 \mu \mathrm{m})$. The rest of the coupling attenuation stems from fiber cutting. This correlates the data that was obtained through SEM imaging (Fig. 3a) in which an uneven cut edge was observed. Cellulose fibers were cut using a razor blade because cellulose fiber cannot be cut with an optical glass fiber cleaver. Thus, an optimal cutting strategy needs to be developed in the future. The attenuation constants of the optical cellulose fibers were significantly higher compared to typical telecommunication glass silica fiber $(0.03 \mathrm{~dB} /$ $\mathrm{km}$ at 1310 and $0.02 \mathrm{~dB} / \mathrm{km}$ at $1550 \mathrm{~nm}$, Corning SMF-28) (Corning 2019), hardcore silica HCS $(5-6 \mathrm{~dB} / \mathrm{km}$ at $820 \mathrm{~nm})$, PMMA $(55 \mathrm{~dB} / \mathrm{km}$ at $538 \mathrm{~nm}$ ) and PS (330 dB/km at $570 \mathrm{~nm}$ ) (Zubia and Arrue 2001). The optical properties of chitosan have been studied using the out-of-plane scattering technique (Jiang et al. 1996). The attenuation constant of chitosan film sandwiched between $\mathrm{SiO}_{2}$ was reported to be $0.5 \mathrm{~dB} / \mathrm{cm}$.
Use of optical cellulose fiber in a water sensor

The use of optical cellulose fiber in a water sensor was indicatively tested. The optical cellulose fiber was positioned to submerge in water and the change in light attenuation was observed (Fig. 6a). About $20 \mathrm{~mm}$ of the fiber was under water. The study clearly shows that the light intensity started decreasing (attenuation increased) when the fiber was kept in water for $10 \mathrm{~min}$ (Fig. 6b). When the fiber was taken out of the water and allowed to dry, the attenuation returned to the zero level within $20 \mathrm{~min}$. Attenuation reached over $30 \mathrm{~dB}$ within the $10 \mathrm{~min}$ swelling in water. Measurement dynamics could be even higher with shorter fibers because a $76 \mathrm{~mm}$-long fiber already has a $48 \mathrm{~dB}$ initial. In addition, higher light intensity will create more measurement dynamics. Moreover, this indicates that both cellulose acetate cladding and pure cellulose core are sensitive to water. Water sensor experiment was repeated with shorter 3 min swelling time (Fig. 6b). In the second measurement attenuation increases $19 \mathrm{~dB}$ and returned to zero level within $10 \mathrm{~min}$. Second swelling and drying velocities are in the same range than within first swelling and drying.

\section{Conclusions}

Optical cellulose fiber was prepared from regenerated cellulose filaments by coating it with cellulose acetate. Optical cellulose fiber has mechanical properties 


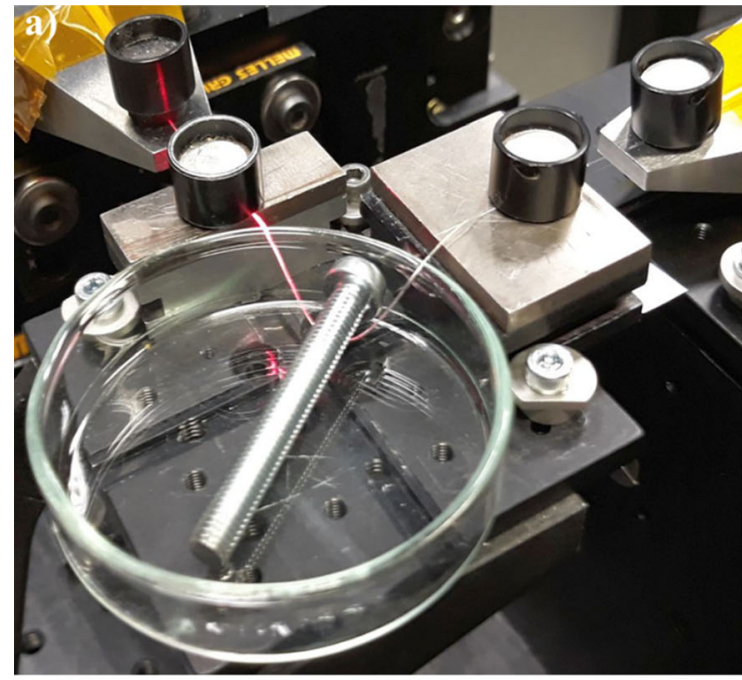

Fig. 6 Test setup to investigate the water sensitivity of optical cellulose fiber (length $76 \mathrm{~mm}$ ) (a). Light attenuation $(\lambda=1300 \mathrm{~nm})$ when the fiber was positioned two times in

similar to the textile fibers previously presented. UVVIS measurements show that pure cellulose is more optically transparent in the visible and IR-wavelength range, as well as screening UV light. Acetylation increases the transparency of cellulose in the UV range. The optical properties of optical cellulose fibers were studied using measurement tools used for optical fibers. The study showed that the attenuation constant of the fiber was $6.3 \mathrm{~dB} / \mathrm{cm}$ at $1300 \mathrm{~nm}$. Use in a water sensor was demonstrated and the attenuation increased reversibly over $30 \mathrm{~dB}$ when the $76 \mathrm{~mm}$-long fiber was placed in water. The optical cellulose fiber could find applications from sensoring, in which the easy modifiability and high temperature resistance are good properties.

Acknowledgments Open access funding provided by Technical Research Centre of Finland (VTT). This work was internally funded by VTT with a government grant and formed part of the Academy of Finland's Flagship Program under Project Nos. 318890 and 318891 (Competence Center for Materials Bioeconomy, FinnCERES).

Author contributions The manuscript was written through contributions from all authors. H.O. was responsible for preparing the project plan, instructed the research work and wrote the first draft of the article. A.H. performed optical fiber measurements and processed the given data. I.L. was responsible for manufacturing cellulose acetate and regenerated cellulose films, as well as conducting UV-VIS, FTIR measurements and processing the given data. A.H and I.L

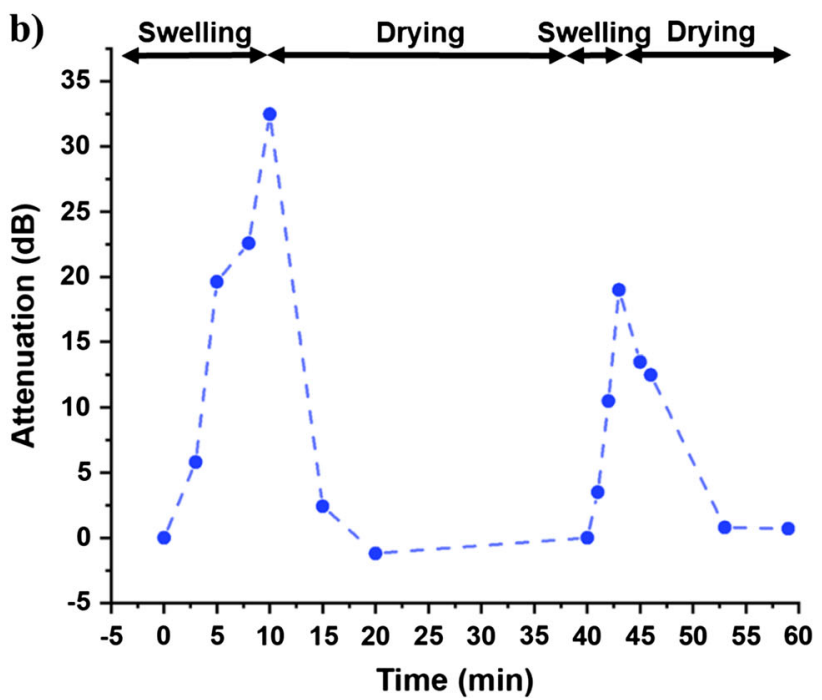

water (0-10 $\mathrm{min}$ and 40-43 $\mathrm{min})$ and subsequent drying in air (10-40 min and 43-60 min) (b)

contribution was equal. K.K. dissolved cellulose and cellulose acetate and prepared optical cellulose fibers. M.K. participated in the optical measurements. A.H. applied for funding for the project and supervised the work. All authors have given their approval to the final version of the manuscript.

\section{Compliance with ethical standards}

Conflict of interest There is no conflict of interest.

Open Access This article is distributed under the terms of the Creative Commons Attribution 4.0 International License (http:// creativecommons.org/licenses/by/4.0/), which permits unrestricted use, distribution, and reproduction in any medium, provided you give appropriate credit to the original author(s) and the source, provide a link to the Creative Commons license, and indicate if changes were made.

\section{References}

Andrade JD, Vanwagenen RA, Gregonis DE et al (1985) Remote fiber-optic biosensors based on evanescent-excited fluoro-immunoassay: concept and progress. IEEE Trans Electron Devices 32:1175-1179

Born M, Wolf E (2013) Principles of optics: electromagnetic theory of propagation, interference and diffraction of light. Elsevier, Amsterdam

Browne MA, Crump P, Niven SJ et al (2011) Accumulation of microplastic on shorelines woldwide: sources and sinks. Environ Sci Technol 45:9175-9179 
Corning (2019) Corning SMF-28 Ultra optical fiber (Product information). In: Prod. Inf. Lett. https://www.corning.com/ media/worldwide/coc/documents/Fiber/SMF-28 Ultra.pdf

Dhadwal HS, Kemp P, Aller J, Dantzler MM (2004) Capillary waveguide nucleic acid based biosensor. Anal Chim Acta 501:205-217. https://doi.org/10.1016/j.aca.2003.09.035

Dupuis A, Guo N, Gao Y et al (2007) Prospective for biodegradable microstructured optical fibers. Opt Lett 32:109-111. https://doi.org/10.1364/OL.32.000109

Hauru LKJ, Hummel M, Michud A, Sixta H (2014) Dry jet-wet spinning of strong cellulose filaments from ionic liquid solution. Cellulose 21:4471-4481. https://doi.org/10.1007/ s10570-014-0414-0

Heinze T, Koschella A (2005) Solvents applied in the field of cellulose chemistry: a mini review. Poli-Meros 15:84-90

Hondros D, Debye P (1910) Elektromagnetische wellen an dielektrischen drähten. Ann Phys 337:465-476

Jiang H, Su W, Caracci S et al (1996) Optical waveguiding and morphology of chitosan thin films. J Appl Polym Sci 61:1163-1171

Kaijanen L, Paakkunainen M, Pietarinen S et al (2015) Ultraviolet detection of monosaccharides: multiple wavelength strategy to evaluate results after capillary zone electrophoretic separation. Int $\mathrm{J}$ Electrochem Sci 10:2950-2961

Klein S, Petersen S, Taylor U et al (2010) Quantitative visualization of colloidal and intracellular gold nanoparticles by confocal microscopy. J Biomed Opt 15:36011-36015

Klemm D, Philipp B, Heinze T (1998) Comprehensive cellulose chemistry, vol 2. Functionalization of cellulose, $\mathrm{VCH}$, Weinheim

Liebert T (2010) Cellulose solvents - remarkable history, bright future. Cellulose solvents: for analysis, shaping and chemical modification. American Chemical Society, Washington, pp 1-3

Liebert TF, Heinze T (2005) Tailored cellulose esters: synthesis and structure determination. Biomacromol 6:333-340

Lindman B, Karlström G, Stigsson L (2010) On the mechanism of dissolution of cellulose. J Mol Liq 156:76-81. https:// doi.org/10.1016/j.molliq.2010.04.016

Luna-Martínez JF, Hernández-Uresti DB, Reyes-Melo ME et al (2011) Synthesis and optical characterization of $\mathrm{ZnS}-$ sodium carboxymethyl cellulose nanocomposite films. Carbohydr Polym 84:566-570. https://doi.org/10.1016/j. carbpol.2010.12.021

Mark JE (2007) Physical properties of polymers handbook. Springer, Berlin

Michud A, Tanttu M, Asaadi S et al (2016) Ioncell-F: ionic liquid-based cellulosic textile fibers as an alternative to viscose and Lyocell. Text Res J 86:543-552

Nazempour R, Zhang Q, Fu R, Sheng X (2018) Biocompatible and implantable optical fibers and waveguides for biomedicine. Mater (Basel, Switzerland) 11:1283. https://doi.org/ 10.3390/ma11081283
Peters K (2011) Polymer optical fiber sensors-a review. Smart Mater Struct 20:013002. https://doi.org/10.1088/0964$1726 / 20 / 1 / 013002$

Ritari T, Tuominen J, Ludvigsen H et al (2004) Gas sensing using air-guiding photonic bandgap fibers. Opt Express 12:4080-4087

Rowen JW, Hunt CM, Plyler EK (1947) Absorption spectra in the detection of chemical changes in cellulose and cellulose derivatives. Text Res J 17:504-511

Sanghera JS, Shaw LB, Aggarwal ID (2002) Applications of chalcogenide glass optical fibers. Comptes Rendus Chim 5:873-883. 0748(02)01450-9

https://doi.org/10.1016/S1631-

Schlamadinger B, Marland G (1996) The role of forest and bioenergy strategies in the global carbon cycle. Biomass Bioenergy 10:275-300. https://doi.org/10.1016/09619534(95)00113-1

Schmalensee R, Stoker TM, Judson RA (1998) World carbon dioxide emissions: 1950-2050. Rev Econ Stat 80:15-27

Schriever O (1920) Elektromagnetische Wellen an dielektrischen Drähten. Ann Phys 368:645-673

Sekimoto S, Nakagawa H, Okazaki S et al (2000) A fiber-optic evanescent-wave hydrogen gas sensor using palladiumsupported tungsten oxide. Sens Actuators B Chem 66:142-145

Senior JM, Jamro MY (2009) Optical fiber communications: principles and practice. Pearson Education, London

Shan D, Zhang C, Kalaba S et al (2017) Flexible biodegradable citrate-based polymeric step-index optical fiber. Biomaterials 143:142-148. https://doi.org/10.1016/j.biomaterials. 2017.08.003

Sindhu KA, Prasanth R, Thakur VK (2014) Medical applications of cellulose and its derivatives: present and future. In: Nanocellulose polymer nanocomposites

Tenhunen T-M, Moslemian O, Kammiovirta K et al (2018) Surface tailoring and design-driven prototyping of fabrics with 3D-printing: an all-cellulose approach. Mater Des 140:409-419. https://doi.org/10.1016/j.matdes.2017.12. 012

Wu DKC, Kuhlmey BT, Eggleton BJ (2009) Ultrasensitive photonic crystal fiber refractive index sensor. Opt Lett 34:322-324

Xu W, Huang W-B, Huang X-G, Yu C (2013) A simple fiberoptic humidity sensor based on extrinsic Fabry-Perot cavity constructed by cellulose acetate butyrate film. Opt Fiber Technol 19:583-586. https://doi.org/10.1016/j.yofte.2013. 09.005

Zubia J, Arrue J (2001) Plastic Optical Fibers: An Introduction to Their Technological Processes and Applications. Opt Fiber Technol 7:101-140. https://doi.org/10.1006/ofte. 2000.0355

Publisher's Note Springer Nature remains neutral with regard to jurisdictional claims in published maps and institutional affiliations. 